Article

\title{
Entropy Generation and Exergy Destruction in Flow of Multiphase Dispersions of Droplets and Particles in a Polymeric Liquid
}

\author{
Rajinder Pal \\ Department of Chemical Engineering, University of Waterloo, Waterloo, ON N2L 3G1, Canada; \\ rpal@uwaterloo.ca; Tel.: +1-519-888-4567 (ext. 32985)
}

Received: 31 December 2017; Accepted: 26 February 2018; Published: 2 March 2018

\begin{abstract}
The theoretical background for entropy generation and exergy destruction in the flow of fluids is reviewed briefly. New experimental results are presented on the quantification of exergy destruction rates in flows of emulsions (oil droplets dispersed in a polymeric liquid), suspensions (solid particles dispersed in a polymeric liquid), and blends of emulsions and suspensions (dispersions of oil droplets and solid particles in a polymeric liquid). A new model is proposed to estimate the exergy destruction rate, and hence power loss, in the flow of multi-phase dispersions of oil droplets, solid particles, and polymeric matrix.
\end{abstract}

Keywords: exergy; entropy; irreversibility; multiphase; dispersion; emulsion; suspension; rheology; non-Newtonian; thermodynamics

\section{Introduction}

Two-phase dispersions (such as oil/water emulsions and solid particles/liquid suspensions) and three-phase dispersions (such as blends of oil droplets, solid particles, and aqueous phase) are encountered in a number of industrial applications [1-10]. A major portion of all the commercial products produced in a modern industrial society are multi-phase dispersions. For example, many food, cosmetic, household, pharmaceutical, and other products of industrial significance, are manufactured and sold in the form of multi-phase mixtures. The manufacturing of these multi-phase products involves mixing, pumping, and flow of multi-phase dispersions in pipelines and other process equipment. Each of these operations are irreversible in nature, and consequently, exergy destruction and power loss occurs in these operations. In order to minimize the power loss and to improve the thermodynamic efficiency of the process, it is important to quantify the irreversibilities in terms of exergy destruction [11,12]. It should be noted that the irreversibility in flow of multi-phase dispersions is due to frictional effects, that is, viscous dissipation of mechanical energy. The viscous dissipation effect in the flow of dispersions is responsible for entropy generation and exergy destruction.

In this paper, new experimental results are presented on rheology and exergy destruction rates in flows of two-phase and three-phase dispersions. The systems investigated are emulsions of oil droplets dispersed in a polymeric liquid, suspensions of solid particles (glass beads) in a polymeric liquid, and three-phase blends of oil droplets, solid particles, and polymeric liquid. The theoretical background necessary for the analysis of exergy destruction in flow of fluids is also reviewed.

This work is in continuation of our earlier studies on the production of entropy and destruction of exergy in flow of dispersions [13-16]. Our earlier studies were restricted to only two-phase oil and water emulsions, where the matrix phase was a Newtonian fluid. This is the first study on the second law analysis (entropy production and exergy destruction) of multi-phase dispersions of droplets and particles suspended in a non-Newtonian polymeric matrix. 


\section{Theoretical Background}

\subsection{Entropy Balance in Fluid Flow}

Entropy is an equilibrium property. It can be expressed as a function of other state variables (such as temperature, volume, pressure, internal energy, etc.), provided that the system is in thermodynamic equilibrium. The state variables do not vary with space and time when the system is in thermodynamic equilibrium. There are no gradients or driving forces present in the system that cause the changes. The fluid under flow condition, however, is not in thermodynamic equilibrium. In general, there are present velocity gradients, temperature gradients, and concentration gradients (if two or more chemical components are present) in fluid, causing transports of momentum, heat, and mass, respectively from one region to another.

Although the bulk fluid under flow conditions is not in thermodynamic equilibrium, it is often necessary to assume that local equilibrium exists everywhere in the fluid. In other words, it is assumed that all the variables defined in equilibrium are defined locally under non-equilibrium condition as well. This is the key hypothesis of classical irreversible thermodynamics (CIT). The CIT assumes that there exists local equilibrium everywhere in the system, and that the state variables vary with space and time. One way to imagine local equilibrium in a system is to subdivide the system into a large number of tiny cells. The size of the cells is selected such that it is large enough for microscopic fluctuations to be negligible, but small enough for local equilibrium to be realized within the individual cells [17]. Although equilibrium exists locally within each cell, the state of equilibrium varies from one cell to another, so that mass, momentum, and energy exchanges are permitted between neighboring cells. Furthermore, the state of equilibrium within a given cell is allowed to vary with time.

Consider an arbitrary volume $V$, fixed in space and bounded by surface $A$. The amount of entropy in that portion of the fluid instantaneously occupying the volume $V$ is given by the integral

$$
S=\int_{V} \rho s d V
$$

where $S$ is the total entropy of the system (fluid) instantaneously occupying the volume $V, \rho$ is the local density of the fluid which may vary from one position to another, and $s$ is the local specific entropy of the fluid. The rate of increase of entropy of the system is the sum of two terms: one term that represents the rate at which entropy enters the system through its bounding surface $A$ due to presence of some gradient, and the other term that represents the rate of production of entropy within the system. Thus, entropy balance over the fluid instantaneously occupying the volume $V$ could be expressed as

$$
\frac{D S}{D t}=\frac{D}{D t} \int_{V} \rho s d V=-\int_{A} \hat{n} \cdot \vec{J}_{s} d A+\int_{V} \dot{\sigma}_{s} d V
$$

where $\hat{n}$ is a unit vector normal to the bounding surface of the system pointing outwardly, $\vec{J}_{S}$ is the entropy flux at the bounding surface of the system $\left(\vec{J}_{S}\right.$ is the rate at which entropy enters the system through the bounding surface per unit area), $\dot{\sigma}_{s}$ is the rate of entropy production within the system per unit volume, and $D / D$ t represents material or substantial time derivative.

Using the Reynolds transport theorem, it can be readily shown that

$$
\frac{D}{D t} \int_{V} \rho s d V=\int_{V} \frac{\partial}{\partial t}(\rho s) d V+\oiint_{A} \hat{n} \cdot \rho \vec{v} s d A
$$

where $\vec{v}$ is the fluid velocity vector. Converting the second integral on the right-hand side of Equation (3) to a volume integral with the help of the Gauss divergence theorem, one can re-write Equation (3) as 


$$
\frac{D}{D t} \int_{V} \rho s d V=\int_{V}\left[\frac{\partial}{\partial t}(\rho s)+\nabla \cdot \rho \vec{v} s\right] d V
$$

Combining Equations (2) and (4) gives

$$
\int_{V}\left[\frac{\partial}{\partial t}(\rho s)+\nabla \cdot \rho \vec{v} s\right] d V=-\int_{A} \hat{n} \cdot \vec{J}_{s} d A+\int_{V} \dot{\sigma}_{s} d V
$$

Converting the first integral on the right-hand side of Equation (5) to a volume integral with the help of the Gauss divergence theorem and rearranging gives

$$
\int_{V}\left[\frac{\partial}{\partial t}(\rho s)+\nabla \cdot \rho \vec{v} s+\nabla \cdot \vec{J}_{s}-\dot{\sigma}_{s}\right] d V=0
$$

Because this equation holds for an arbitrary volume $V$, the integrand must be zero. Thus,

$$
\frac{\partial}{\partial t}(\rho s)+\nabla \cdot \rho \vec{v} s=-\nabla \cdot \vec{J}_{s}+\dot{\sigma}_{s}
$$

Using the following continuity (mass balance) equation along with the definition of the substantial derivative given below,

$$
\begin{aligned}
& \frac{\partial \rho}{\partial t}+\nabla \cdot \rho \vec{v}=0 \\
& \frac{D}{D t}=\frac{\partial}{\partial t}+\vec{v} \cdot \nabla
\end{aligned}
$$

it can be readily shown that

$$
\frac{\partial}{\partial t}(\rho s)+\nabla \cdot \rho \vec{v} s=\rho \frac{D s}{D t}
$$

Combining Equations (7) and (10), it follows that

$$
\rho \frac{D s}{D t}=-\nabla \cdot \vec{J}_{s}+\dot{\sigma}_{s}
$$

According to the second law of thermodynamics, $\dot{\sigma}_{s} \geq 0$, and therefore,

$$
\dot{\sigma}_{s}=\rho \frac{D s}{D t}+\nabla \cdot \vec{J}_{s} \geq 0
$$

The "greater than" sign in the above relation is valid for real (irreversible) processes and the "equal to" sign is valid for reversible processes.

\subsection{Momentum and Energy Balance Equations for Fluid Flow}

According to Newton's second law of motion, the time rate of change of linear momentum of a fluid instantaneously occupying an arbitrary volume, $V$, fixed in space, is equal to the sum of forces acting upon the fluid occupying $V$. This principle leads to the following equation of motion for fluids [18]:

$$
\rho \frac{D \vec{v}}{D t}=\nabla \cdot \underline{\underline{\pi}}+\rho \vec{g}
$$

where $\vec{g}$ is acceleration due to gravity and $\underline{\underline{\pi}}$ is total stress tensor that can be expressed as

$$
\underline{\underline{\pi}}=-p \underline{\underline{\delta}}+\underline{\underline{\tau}}
$$


where $p$ is the local thermodynamic pressure, $\underline{\underline{\delta}}$ is the unit tensor, and $\underline{\underline{\tau}}$ is the extra or deviatoric stress tensor (often referred to as viscous stress tensor). Upon substitution of the expression for $\underline{\underline{\pi}}$ from Equation (14) into Equation (13), the equation of motion becomes:

$$
\rho \frac{D \vec{v}}{D t}=-\nabla p+\nabla \cdot \underline{\underline{\tau}}+\rho \vec{g}
$$

Applying the first law of thermodynamics (energy balance) to fluid flow, the following total energy equation for fluids can be obtained:

$$
\rho \frac{D e}{D t}=-\nabla \cdot \vec{q}^{\prime \prime}+\nabla \cdot \underline{\underline{\pi}} \cdot \vec{v}=-\nabla \cdot \vec{q}^{\prime \prime}-\nabla \cdot p \vec{v}+\nabla \cdot \underline{\underline{\tau}} \cdot \vec{v}
$$

where $e$ is the specific total energy of the fluid and $\vec{q}^{\prime \prime}$ is the heat flux.

The mechanical energy equation can be obtained from the equation of motion (Equation (15)) by taking the scalar (dot) product between Equation (15) and the fluid velocity vector as

$$
\rho \frac{D}{D t}\left(v^{2} / 2\right)=-\vec{v} \cdot \nabla p+\vec{v} \cdot \nabla \cdot \underline{\underline{\tau}}+\rho \vec{v} \cdot \vec{g}
$$

where $v$ is the magnitude of the velocity vector $\vec{v}$. Upon subtraction of the mechanical energy equation, Equation (17), from the total energy equation, Equation (16), the following internal energy equation (also referred to as thermal energy equation) is obtained:

$$
\rho \frac{D u}{D t}=-\nabla \cdot \vec{q}^{\prime \prime}-p \nabla \cdot \vec{v}+\underline{\underline{\tau}}: \nabla \vec{v}
$$

where $u$ is the specific internal energy of the fluid.

\subsection{Application of the Gibbs Equation to Fluid Flow}

For a single-component fluid (pure substance), the relationship between entropy and other state variables is expressed by the Gibbs equation given below:

$$
d s=T^{-1} d u+p T^{-1} d v
$$

where $T$ is the absolute temperature, and $v$ is the specific volume of fluid. Strictly speaking, this relationship is valid for a system in thermodynamic equilibrium. It can be used to calculate entropy changes when the system traverses from one equilibrium state to another. However, in classical irreversible thermodynamics (CIT), it is assumed that the local state variables are related to each other by the same equations of state as in equilibrium. Thus, the Gibbs relation (Equation (19)) can be applied to systems locally, even though the systems are in non-equilibrium state.

The Gibbs relation, Equation (19), could be re-written in terms of rates as follows:

$$
\frac{D s}{D t}=T^{-1} \frac{D u}{D t}+p T^{-1} \frac{D v}{D t}
$$

At any given point in the flow field, this equation describes the time rate of change of specific entropy of a fluid element instantaneously located at that point. Combining Equations (18) and (20), the following relation is obtained:

$$
\rho \frac{D s}{D t}=T^{-1}\left[-\nabla \cdot \vec{q}^{\prime \prime}-p \nabla \cdot \vec{v}+\underline{\underline{\tau}}: \nabla \vec{v}\right]+\rho p T^{-1} \frac{D v}{D t}
$$

Using the second law of thermodynamics (Equation (12)) and Equation (21), it follows that 


$$
\dot{\sigma}_{s}=T^{-1}\left[-\nabla \cdot \vec{q}^{\prime \prime}-p \nabla \cdot \vec{v}+\underline{\underline{\tau}}: \nabla \vec{v}\right]+\rho p T^{-1} \frac{D v}{D t}+\nabla \cdot \vec{J}_{s} \geq 0
$$

Using the continuity equation (Equation (8)), Equation (22) further simplifies to

$$
\dot{\sigma}_{s}=T^{-1}\left[-\nabla \cdot \vec{q}^{\prime \prime}+\underline{\underline{\tau}}: \nabla \vec{v}\right]+\nabla \cdot \vec{J}_{s} \geq 0
$$

In a single-component fluid (pure substance), the entropy flux $\vec{J}_{S}$ is due to temperature gradient in the fluid. The entropy flux $\vec{J}_{s}$ is related to the heat flux $\vec{q}^{\prime \prime}$ as follows

$$
\vec{J}_{s}=\frac{\bar{q}^{\prime \prime}}{T}
$$

Using Equations (23) and (24), the rate of entropy production per unit volume of fluid can be expressed as

$$
\dot{\sigma}_{s}=\vec{q}^{\prime \prime} \cdot \nabla\left(T^{-1}\right)+T^{-1}(\underline{\underline{\tau}}: \nabla \vec{v}) \geq 0
$$

Thus, the rate of entropy production in flow of single-component fluids (or multi-component fluids of uniform composition, without any concentration gradients) is due to two mechanisms: irreversible heat transfer caused by temperature gradient, and viscous dissipation of mechanical energy caused by velocity gradient. In the absence of temperature gradients in the fluid, Equation (25) reduces to

$$
\dot{\sigma}_{s}=T^{-1}(\underline{\underline{\tau}}: \nabla \vec{v}) \geq 0
$$

\subsection{Exergy Destruction in Fluid Flow}

The exergy of a fluid per unit mass $(\psi)$ is given as

$$
\psi=\left(h-h_{o}\right)-T_{o}\left(s-s_{o}\right)+\frac{v^{2}}{2}+g z
$$

where $h$ is the specific enthalpy of fluid, $h_{0}$ and $s_{o}$ are specific enthalpy and specific entropy of fluid in the dead state, respectively, $T_{o}$ is the absolute temperature of the environment (surroundings), $v$ is the magnitude of velocity vector of fluid, $g$ is the magnitude of acceleration due to gravity, and $z$ is the elevation of the fluid stream with respect to the dead state. In this expression of exergy, it is assumed that the chemical composition of the fluid is constant.

Using the Gouy-Stodola theorem of thermodynamics [12], one can calculate exergy destruction in flow of fluids as

$$
\dot{\psi}_{D}=T_{o} \dot{\sigma}_{s}=T_{o} T^{-1}(\underline{\underline{\tau}}: \nabla \vec{v}) \geq 0
$$

where $\dot{\psi}_{D}$ is the rate of exergy destruction per unit volume during the flow of fluid.

\section{Experimental Work}

\subsection{Experimental Set Up}

In this work, the entropy generation, and hence exergy destruction, in torsional flow of multiphase dispersions is investigated experimentally using a parallel plate geometry. The multiphase dispersions consisted of oil droplets and solid particles (glass beads) dispersed in a continuum of polymeric matrix fluid.

Figure 1 shows a schematic diagram of the parallel plate geometry used in the experiments. The fluid (multiphase dispersion) is placed in the gap between the parallel plates. The upper plate is rotated at an angular velocity $\Omega$, and the bottom plate is kept stationary. The torque required to rotate the upper plate at $\Omega$ is measured. 


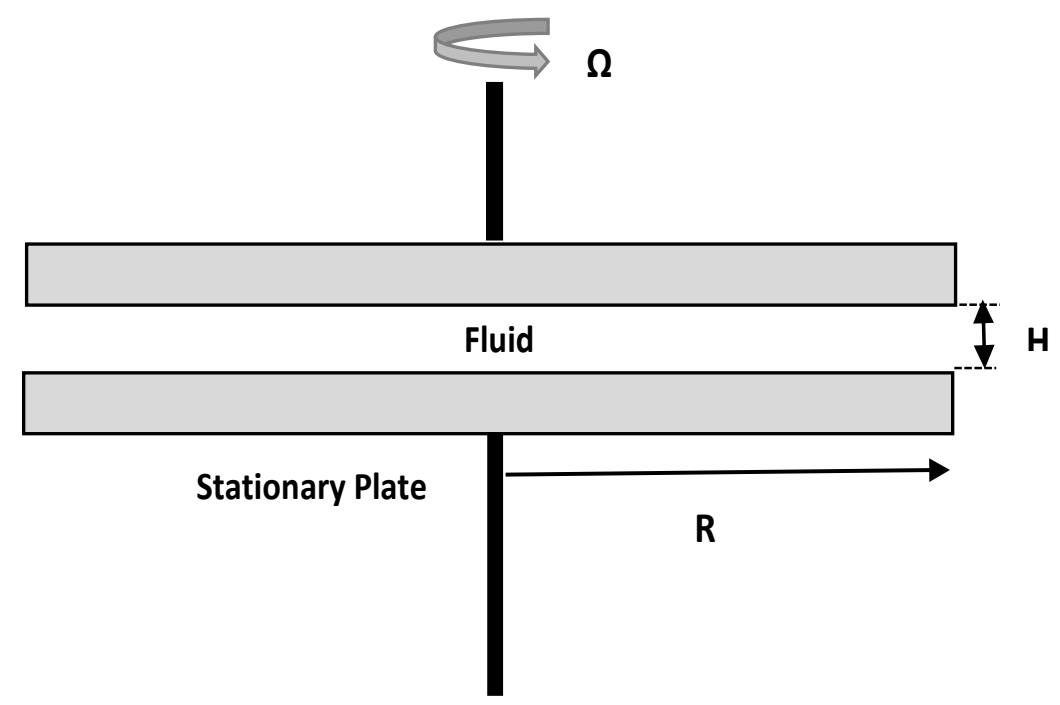

Figure 1. Schematic diagram of a parallel plate geometry.

In order to calculate the rate of exergy destruction or the rate of entropy production, the term $\underline{\underline{\tau}}: \nabla \vec{v}$ is needed. In cylindrical coordinates, $\underline{\underline{\tau}}: \nabla \vec{v}$ is given as [19]

$$
\begin{aligned}
& \underline{\underline{\tau}}: \nabla \vec{v}=\tau_{r r}\left(\frac{\partial v_{r}}{\partial r}\right)+\tau_{r \theta}\left(\frac{1}{r} \frac{\partial v_{r}}{\partial \theta}-\frac{v_{\theta}}{r}\right)+\tau_{r z}\left(\frac{\partial v_{r}}{\partial z}\right)+\tau_{\theta r}\left(\frac{\partial v_{\theta}}{\partial r}\right)+\tau_{\theta \theta}\left(\frac{1}{r} \frac{\partial v_{\theta}}{\partial \theta}+\frac{v_{r}}{r}\right) \\
& +\tau_{\theta z}\left(\frac{\partial v_{\theta}}{\partial z}\right)+\tau_{z r}\left(\frac{\partial v_{z}}{\partial r}\right)+\tau_{z \theta}\left(\frac{1}{r} \frac{\partial v_{z}}{\partial \theta}\right)+\tau_{z z}\left(\frac{\partial v_{z}}{\partial z}\right)
\end{aligned}
$$

The velocity distribution in the gap between the parallel plates of Figure 1 is given as [19]

$$
v_{\theta}=\Omega \frac{r z}{H}
$$

where $r$ is the radial position coordinate from the origin fixed at the center of the bottom plate, and $z$ is the vertical position coordinate from the origin. Using the above velocity distribution, Equation (29) reduces to

$$
\underline{\underline{\tau}}: \nabla \vec{v}=\tau_{\theta z}\left(\frac{\Omega r}{H}\right)
$$

The shear rate $(\dot{\gamma})$ at any radial location is given as $[19,20]$

$$
\dot{\gamma}=\Omega \frac{r}{H}
$$

Thus,

$$
\underline{\underline{\tau}}: \nabla \vec{v}=\tau_{z \theta} \dot{\gamma}
$$

Thus, the rate of energy dissipation in frictional heating of the fluid per unit volume of fluid, that is, the term $\underline{\underline{\tau}}: \nabla \vec{v}$, at any location in the gap between the parallel plates can be determined from the knowledge of shear stress $\tau_{z \theta}$ and shear rate $\dot{\gamma}$ at that location. Note that the term $\underline{\underline{\tau}}: \nabla \vec{v}$ is always positive, and that the stress tensor $\underline{\underline{\tau}}$ is symmetric. In the present work, Bohlin controlled-stress rheometer (Bohlin CS-50) was used with a parallel plate geometry. The parallel plate geometry consisted of a stainless steel upper plate (diameter $25 \mathrm{~mm}$ ) and an aluminum lower plate (diameter $40 \mathrm{~mm}$ ). The gap width between the plates was kept at $1 \mathrm{~mm}$. The rheological measurements were carried out at $25{ }^{\circ} \mathrm{C}$. From the measurement of the torque required to rotate the upper plate at $\Omega$, the shear stress $\tau_{z \theta}$ is determined at the edge of the upper plate, where the shear rate is given as $\dot{\gamma}=\Omega R / H[19,20]$. 


\subsection{Preparation of Multiphase Dispersions}

The multiphase dispersions of droplets and particles in a polymeric liquid were prepared by blending emulsions and suspensions gently in different proportions, while keeping the total volume fraction of the dispersed phase $(\phi)$ the same. The emulsions and suspensions were prepared individually with the same dispersion medium (aqueous polymer-surfactant solution) using a Gifford-Wood homogenizer. The dispersion medium of emulsions and suspensions and their blends consisted of $1.02 \mathrm{wt} \%$ polymer and $2 \mathrm{wt} \%$ surfactant dispersed in deionized water. The polymer used was Hercules cellulose gum (type 7H4F PM), which is purified sodium carboxymethyl cellulose. The molecular weight of the polymer was $7 \times 10^{5}$ approximately. The cellulose gum is an anionic water-soluble polymer used in a number of applications in various industries. The surfactant used was Triton X-100, which is a non-ionic water-soluble surfactant supplied by Union Carbide. The oil used in the preparation of emulsions was odorless kerosene supplied by Fisher Scientific. The viscosity of the oil was $1.5 \mathrm{mPa} \cdot \mathrm{s}$ at $25^{\circ} \mathrm{C}$. The emulsions prepared were of oil-in-water $(\mathrm{O} / \mathrm{W})$ type consisting of oil droplets dispersed in the aqueous polymer-surfactant dispersion medium. The glass beads used in the preparation of suspensions were supplied by Flex-O-Lite Inc. (Crestwood, MO, USA). The Sauter mean diameters of glass beads and oil droplets were $92 \mu \mathrm{m}$ and $3.45 \mu \mathrm{m}$, respectively. The typical photomicrographs of glass beads and oil droplets are shown in Figure 2. The emulsions and suspensions, and their blends, were prepared with four different volume fractions of the dispersed phase: $\phi=0.176, \phi=0.289, \phi=0.377$, and $\phi=0.449$.

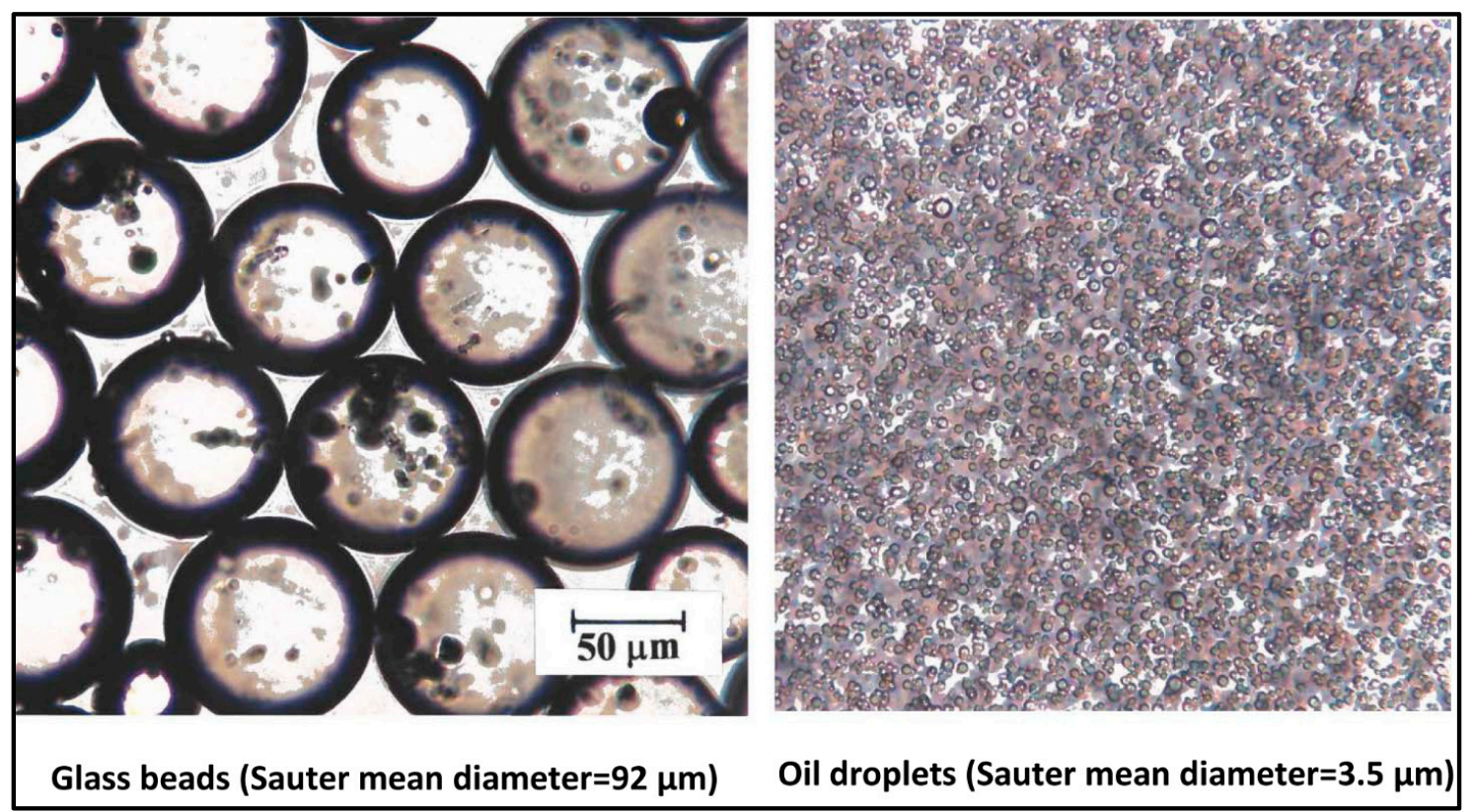

Figure 2. Sample photomicrographs of glass beads (suspension) and oil droplets (emulsion).

\section{Results and Discussion}

\subsection{Two-Phase Dispersions}

\subsubsection{Rheology of Two-Phase Dispersions}

Figure 3 shows the shear stress versus shear rate plots of suspensions of spherical glass beads in polymeric matrix. The experimental data for suspensions with different concentrations of solid particles follow a power-law behavior described by the following equation:

$$
\tau=K \dot{\gamma}^{n}
$$


where $K$ is the consistency index and $n$ is the flow behavior index. According to the power-law model, Equation (34), the shear stress versus shear rate plots are linear on a $\log -\log$ scale, with slopes of $n$. Table 1 summarizes the statistical information regarding the power-law fit of shear stress versus shear rate data for suspensions of glass beads in a polymeric matrix.

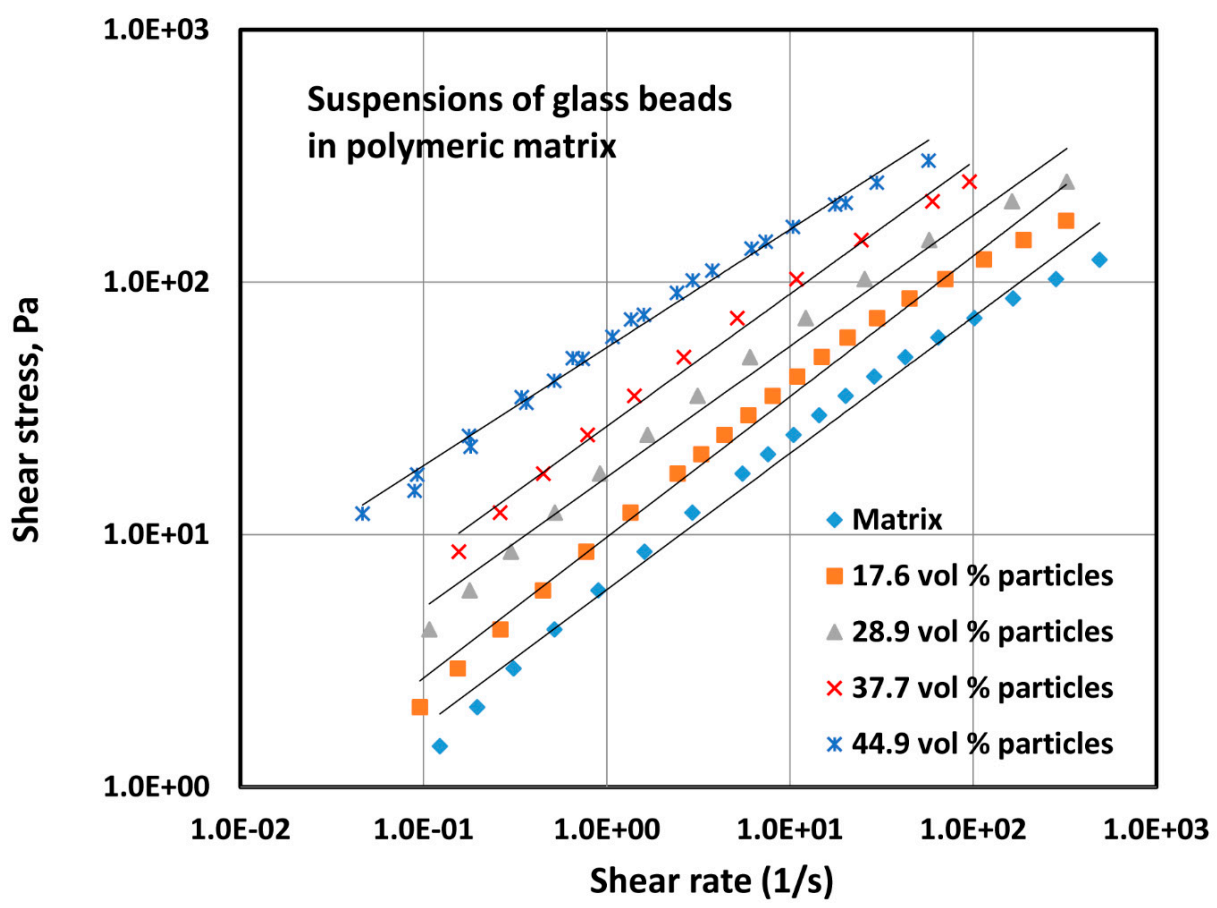

Figure 3. Shear stress versus shear rate plots of suspensions of glass beads in polymeric solution.

Table 1. Goodness of fit of power-law model and confidence intervals for the power-law parameters related to suspensions of glass beads in a polymeric matrix.

\begin{tabular}{cccccc}
\hline $\begin{array}{c}\text { Volume } \\
\text { Percent of } \\
\text { Glass Particles }\end{array}$ & $\begin{array}{c}\text { Regression } \\
\text { Correlation } \\
\text { Coefficient, } \boldsymbol{R}^{\mathbf{2}}\end{array}$ & $\begin{array}{c}\text { Flow Behavior } \\
\text { Index, } \boldsymbol{n}\end{array}$ & $\begin{array}{c}\text { Consistency } \\
\text { Index, } \boldsymbol{K} \\
\text { (Units of Pa.s }{ }^{\boldsymbol{n}} \text { ) }\end{array}$ & $\begin{array}{c}\mathbf{9 5 \%} \\
\text { Confidence } \\
\text { Interval of } \boldsymbol{n}\end{array}$ & $\begin{array}{c}\mathbf{9 5 \%} \\
\text { Confidence } \\
\text { Interval of } \boldsymbol{K}\end{array}$ \\
\hline 0 & 0.9863 & 0.540 & 6.05 & {$[0.507,0.573]$} & {$[5.45,6.73]$} \\
17.6 & 0.9884 & 0.557 & 9.76 & {$[0.527,0.587]$} & {$[8.94,10.58]$} \\
28.9 & 0.9866 & 0.518 & 16.91 & {$[0.479,0.558]$} & {$[15.10,18.94]$} \\
37.7 & 0.9908 & 0.524 & 26.85 & {$[0.488,0.560]$} & {$[24.61,29.26]$} \\
44.9 & 0.9913 & 0.468 & 55.09 & {$[0.452,0.484]$} & {$[53.36,56.88]$} \\
\hline
\end{tabular}

Interestingly, the suspension plots with different volume fractions of the dispersed phase (glass beads) are nearly parallel to each other, indicating that the flow behavior index $n$ does not change appreciably with the introduction of particles in the polymeric matrix. The average flow behavior index is 0.52 , indicating that the suspensions and the matrix phase are pseudoplastic non-Newtonian fluids. However, the suspensions become more viscous with the increase in the particle concentration, as reflected in the increase in the $K$ value (upward shift in the shear stress versus shear rate plots).

Figure 4 shows the shear stress versus shear rate data for $\mathrm{O} / \mathrm{W}$ emulsions with different volume concentrations of oil droplets. The same data are also shown as viscosity versus shear rate in Figure 5 . Emulsions of oil droplets behave very differently from the suspensions of rigid particles. The emulsion data for different oil concentrations closely matches the matrix behavior. Thus, the addition of oil droplets to the polymeric matrix does not cause any appreciable change in the consistency ( $K$ value) and flow behavior ( $n$ value). This is not surprising as emulsion droplets are deformable in nature, and undergo internal circulation when subjected to shear flow. The increase in flow resistance due to the 
introduction of interfaces in emulsions is balanced by the low viscosity of dispersed phase (oil) as compared with the matrix fluid. All the emulsion data could be described by the power-law model (Equation (34)) with $K=6.84 \mathrm{~Pa}^{n} \mathrm{~s}^{n}$ and $n=0.52$. The $95 \%$ confidence intervals of $K$ and $n$ are as follows $[6.53,7.16]$ and $[0.506,0.533]$, respectively.

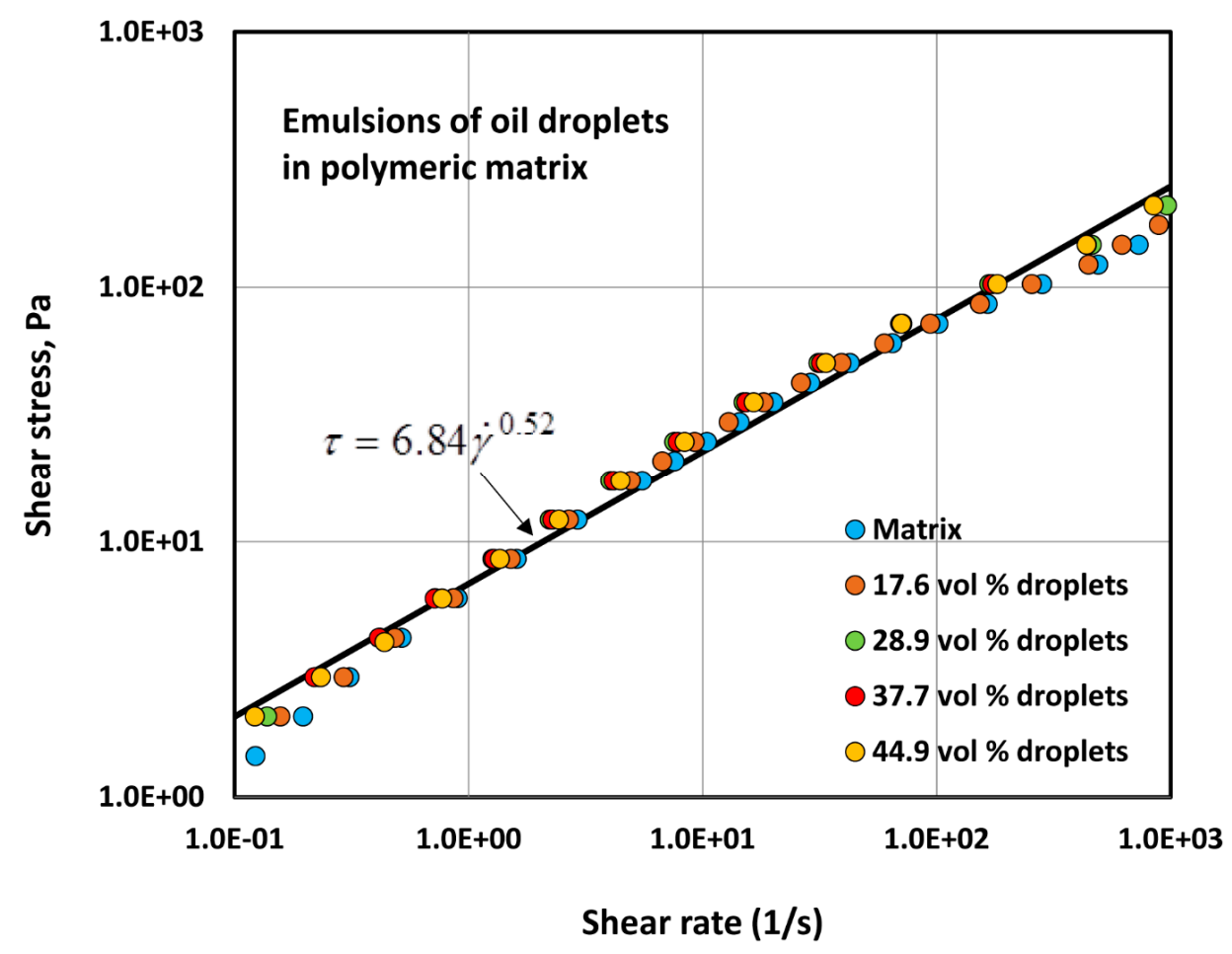

Figure 4. Shear stress versus shear rate data of emulsions of oil droplets in polymeric solution.

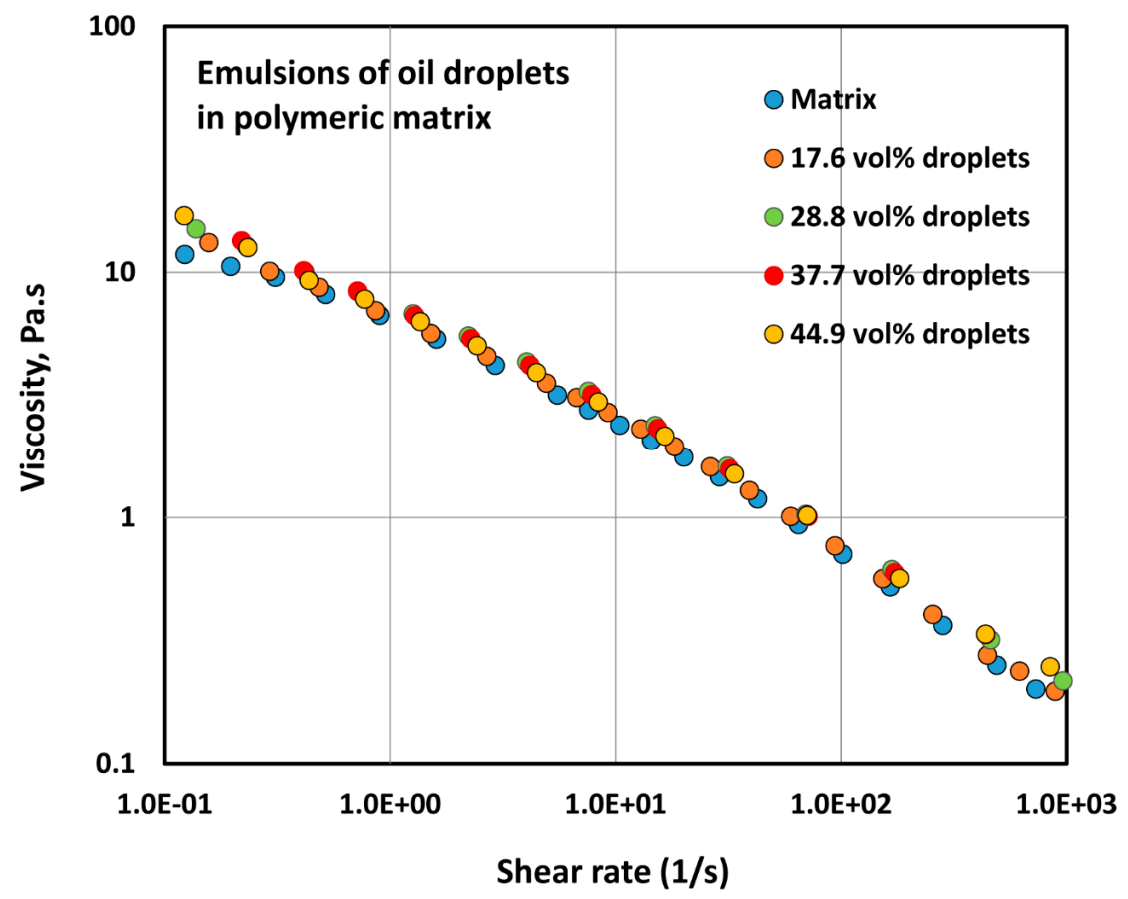

Figure 5. Apparent viscosity versus shear rate data of emulsions of oil droplets in polymeric solution. 
Figure 6 compares the power-law parameters of suspensions and emulsions. The figure reveals the following points: (a) the flow behavior index $n$ of emulsions and suspensions does not differ from that of the polymeric matrix; (b) the consistency index $K$ of emulsions is nearly constant with respect to oil concentration variation; (c) the consistency index of suspensions rises sharply with the increase in the particle concentration; and (d) the suspensions are much more viscous than the emulsions as reflected in the values of the consistency index.

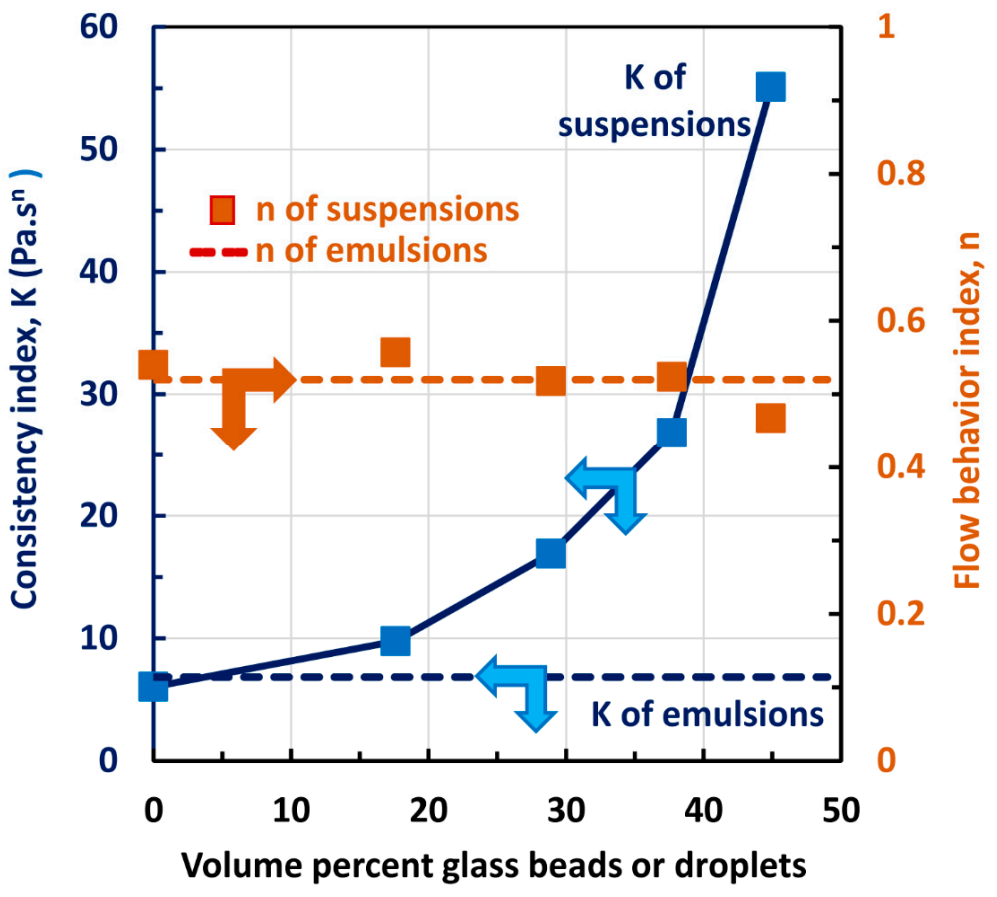

Figure 6. Comparison of power-law parameters of suspensions and emulsions.

\subsubsection{Exergy Destruction in Flow of Two-Phase Dispersions}

From Equations (28) and (33), it follows that the rate of exergy destruction per unit volume of the fluid is given as

$$
\dot{\psi}_{D}=\tau \dot{\gamma}
$$

Note that in our experiments $T=T_{o}=298.15 \mathrm{~K}$. Using the shear stress versus shear rate data obtained from the rheometer, the rate of exergy destruction per unit volume of the fluid was calculated as a function of shear rate from Equation (35).

Figure 7 shows the plots of $\dot{\psi}_{D}$ versus $\dot{\gamma}$ for suspensions of glass particles in polymeric matrix. The plots of exergy destruction rate for suspensions with different volume fractions of the dispersed phase (glass beads) are nearly parallel to each other, as expected from the shear stress versus shear rate plots of Figure 3. Note that Equation (35) in conjunction with the power law model yields

$$
\dot{\psi}_{D}=K \dot{\gamma}^{n+1}
$$

Thus, the slope of the exergy destruction rate plot is simply $(n+1)$. The average value of the slopes of different lines shown in Figure 7 is 1.52 (average value of $n$ was 0.52 , as noted earlier). At any given shear rate, the exergy destruction rate increases with the increase in the particle concentration.

The $\dot{\psi}_{D}$ versus $\dot{\gamma}$ data for emulsions of oil droplets in polymeric matrix are shown in Figure 8 for different concentrations of oil droplets. The data for the emulsions and matrix overlap with each other indicating that for a given shear rate, the exergy destruction rate is independent of the oil droplet concentration. This is because the consistency of emulsions, and hence viscous dissipation of 
mechanical energy in emulsions, does not vary to any appreciable extent with the increase in the oil concentration at any given shear rate.

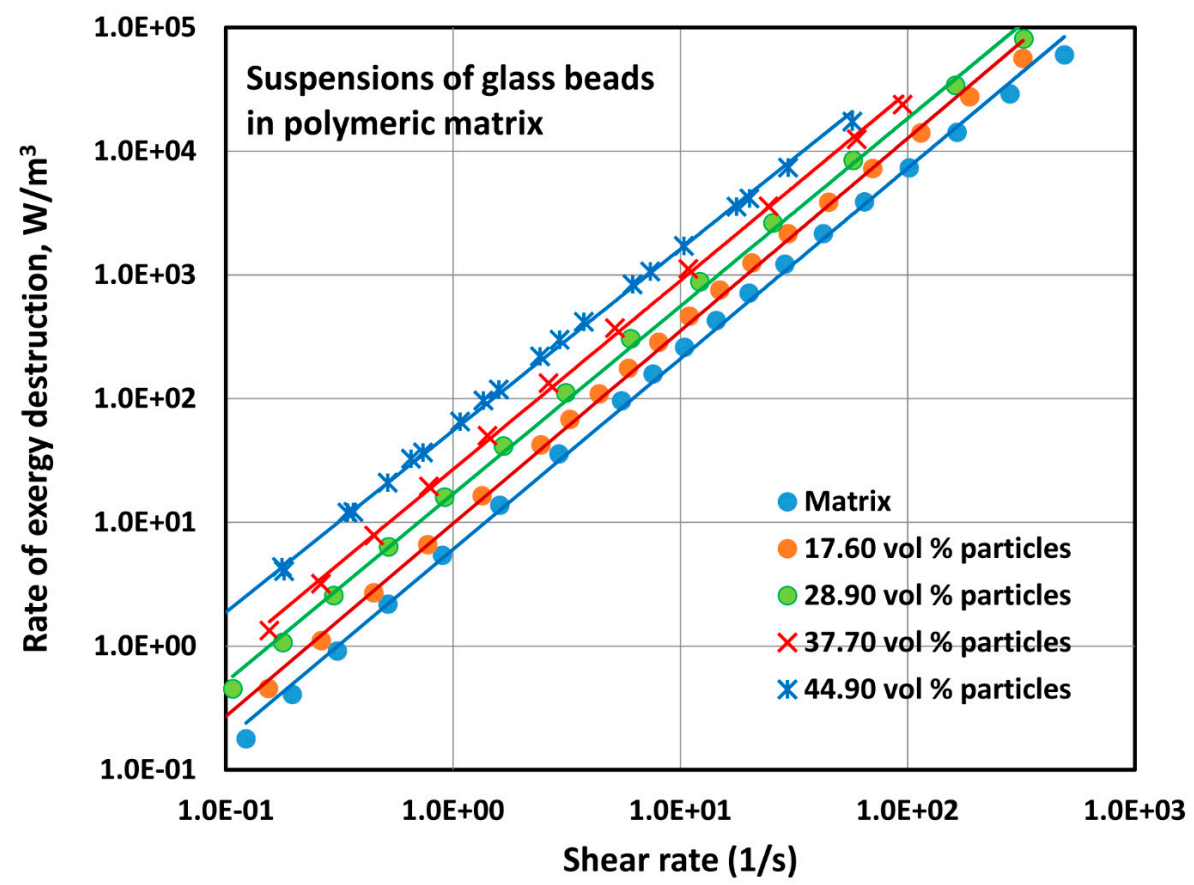

Figure 7. Plots of exergy destruction rates in suspensions of glass beads in polymeric matrix.

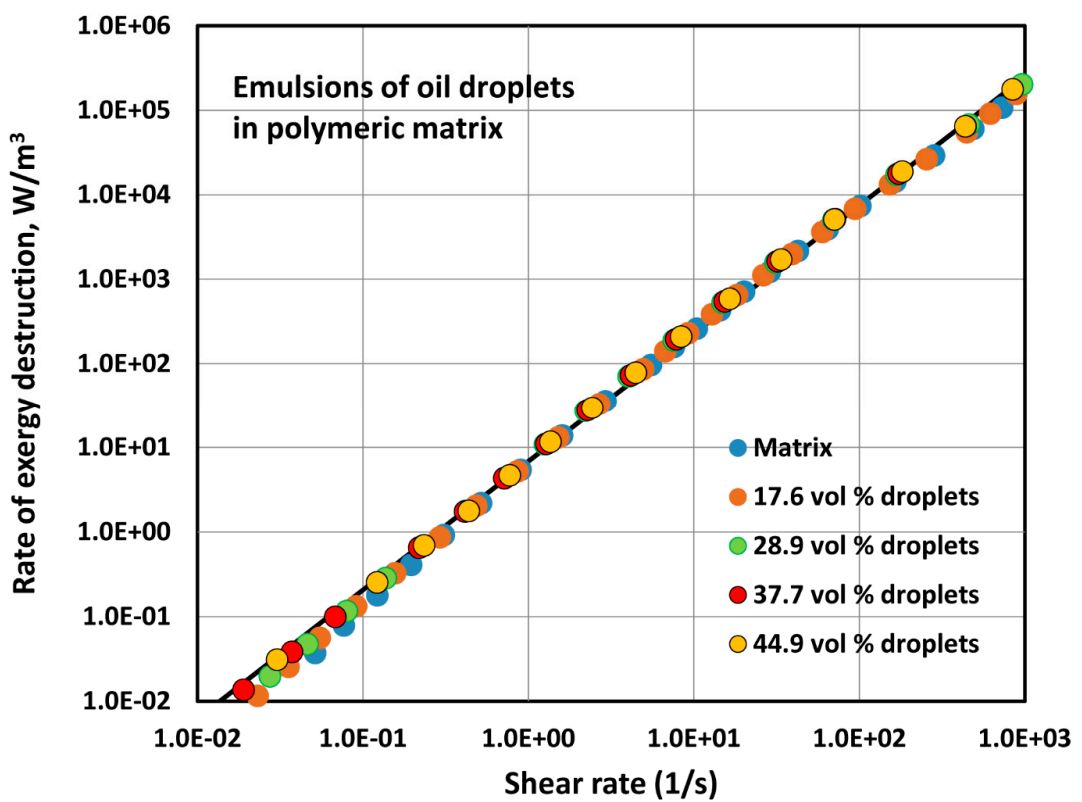

Figure 8. Exergy destruction rate data for emulsions of oil droplets in polymeric matrix.

\subsection{Multi-Phase Dispersions of Oil and Water in a Polymeric Matrix}

\subsubsection{Exergy Destruction in Multi-Phase Dispersions}

As noted earlier, the multiphase dispersions of droplets and particles in a polymeric liquid were prepared by blending emulsions and suspensions gently in different proportions, while keeping the total volume fraction of the dispersed phase $(\phi)$ the same. Figures 9 and 10 show the typical 
exergy destruction behavior of blends of emulsions and suspensions at given total volume fractions of dispersed phase. The total volume percent of the dispersed phase (oil droplets + glass beads) is $37.7 \%$ in Figure 9, and 44.9\% in Figure 10. Upon dilution of the suspension with emulsion of the same dispersed-phase concentration, the exergy destruction rate decreases. The data for blends, with different proportions of emulsion in the blends, fall in between the lines for pure suspension and pure emulsion. However, the variation in the exergy destruction rate with the increase in the proportion of emulsion, keeping total volume fraction fixed, is not linear. This can be seen more clearly in Figures 11 and 12, where the exergy destruction rate is plotted as a function of emulsion content of the blend of emulsion and suspension, keeping the total volume fraction of the dispersed phase (glass particles and oil droplets) and shear rate fixed. The experimental data show negative deviation from the linear behavior.

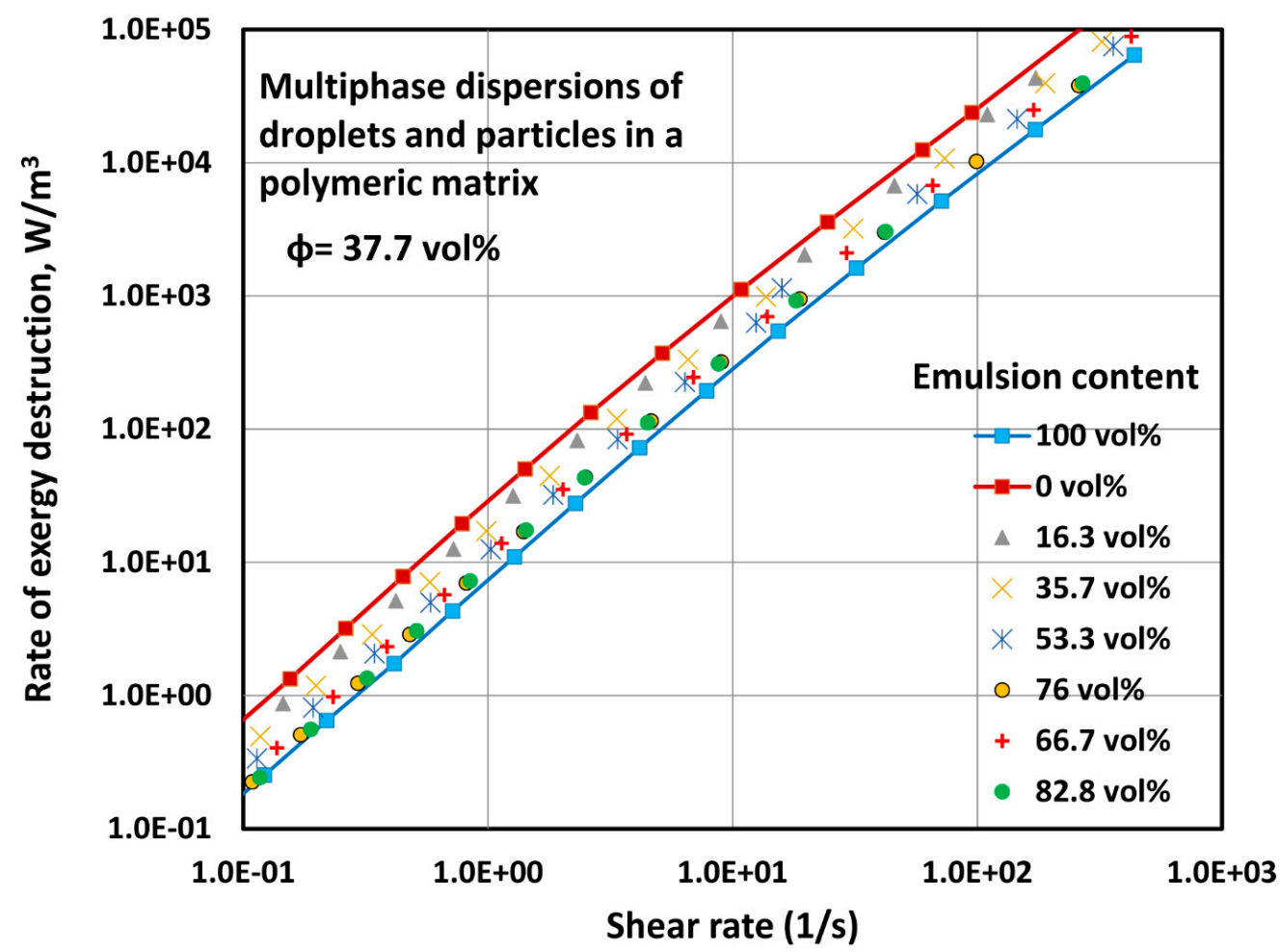

Figure 9. Exergy destruction rate in blends of emulsion and suspension at a constant total volume fraction of dispersed phase of 0.377 .

The exergy destruction rate as a function of the total volume fraction of the dispersed phase (glass beads + oil droplets) is plotted in Figure 13 for different contents of emulsion in the blend of emulsion and suspension, at a fixed shear rate of $10 \mathrm{~s}^{-1}$. As the suspension is diluted with an emulsion having the same volume fraction of the dispersed phase, the exergy destruction rate decreases. In other words, the substitution of solid particles with oil droplets, keeping the total volume fraction of dispersed phase constant, results in a decrease in the exergy destruction rate. This is not unexpected, as the emulsion is relatively much less viscous than the suspension at the same value of the dispersed phase concentration. Also note that for a given content of emulsion in the blend, the exergy destruction rate of the blend increases with the increase in the total volume fraction of the dispersed phase. 


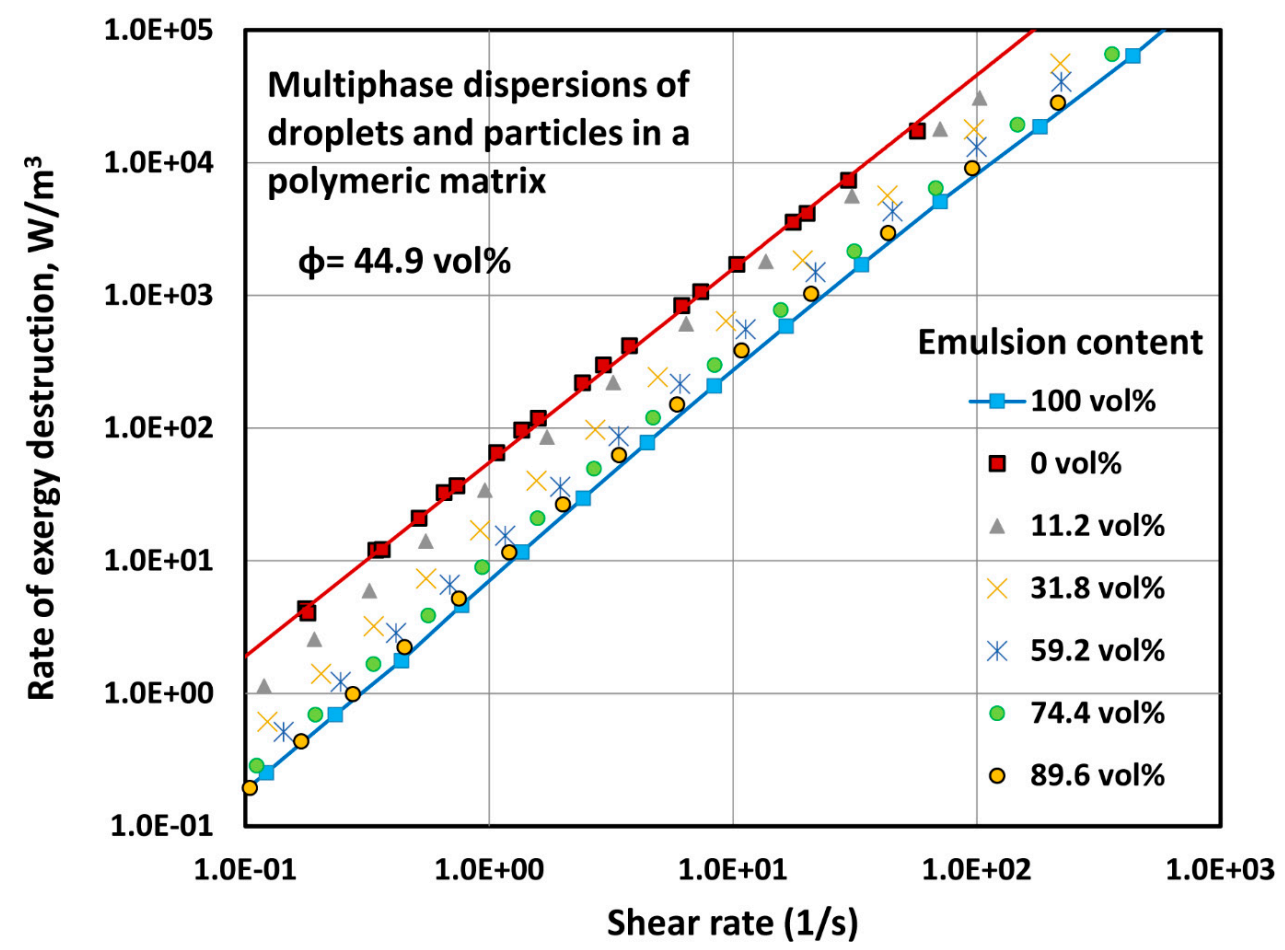

Figure 10. Exergy destruction rate in blends of emulsion and suspension at a constant total volume fraction of dispersed phase of 0.449 .

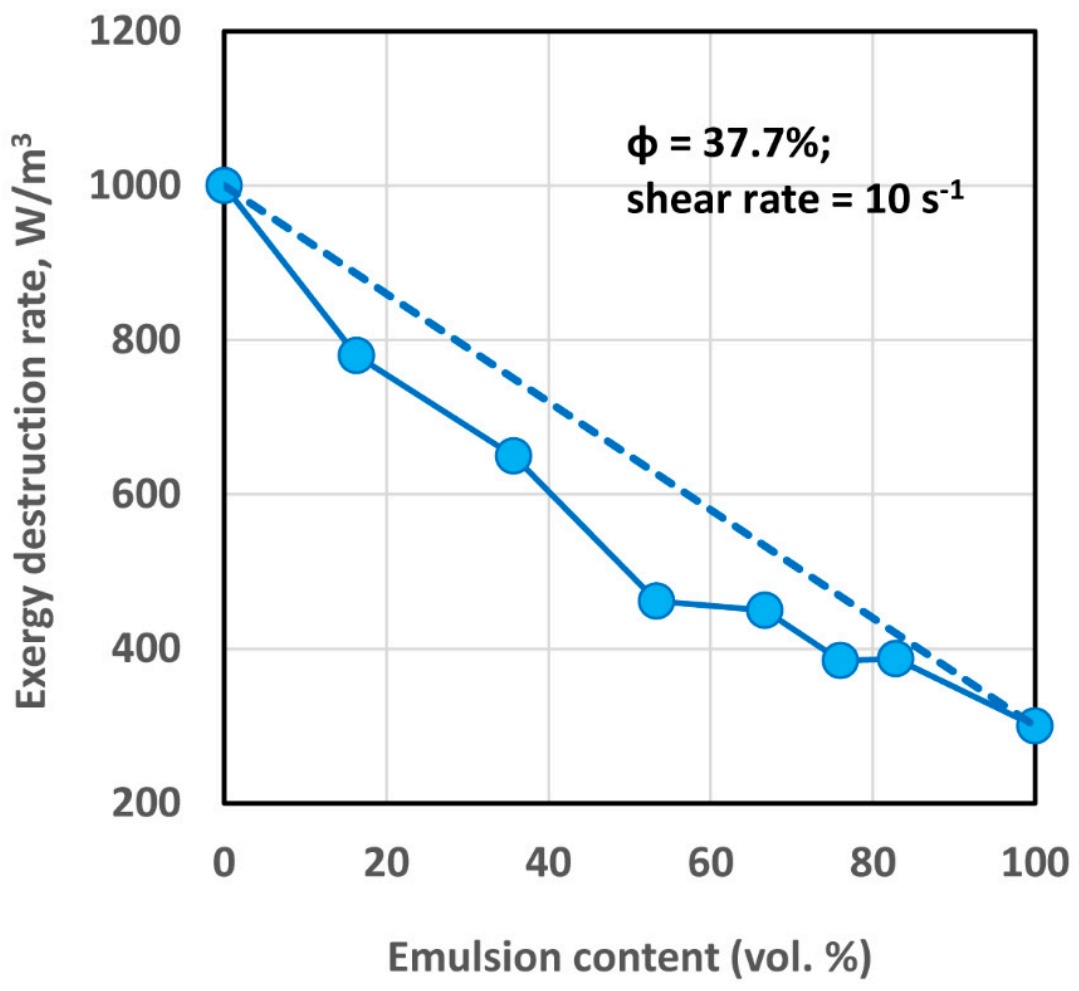

Figure 11. Exergy destruction in blends of emulsion and suspension as a function of emulsion content ( $\mathrm{vol} \%$ ) at a constant total volume fraction of dispersed phase of 0.377 and shear rate of $10 \mathrm{~s}^{-1}$. 


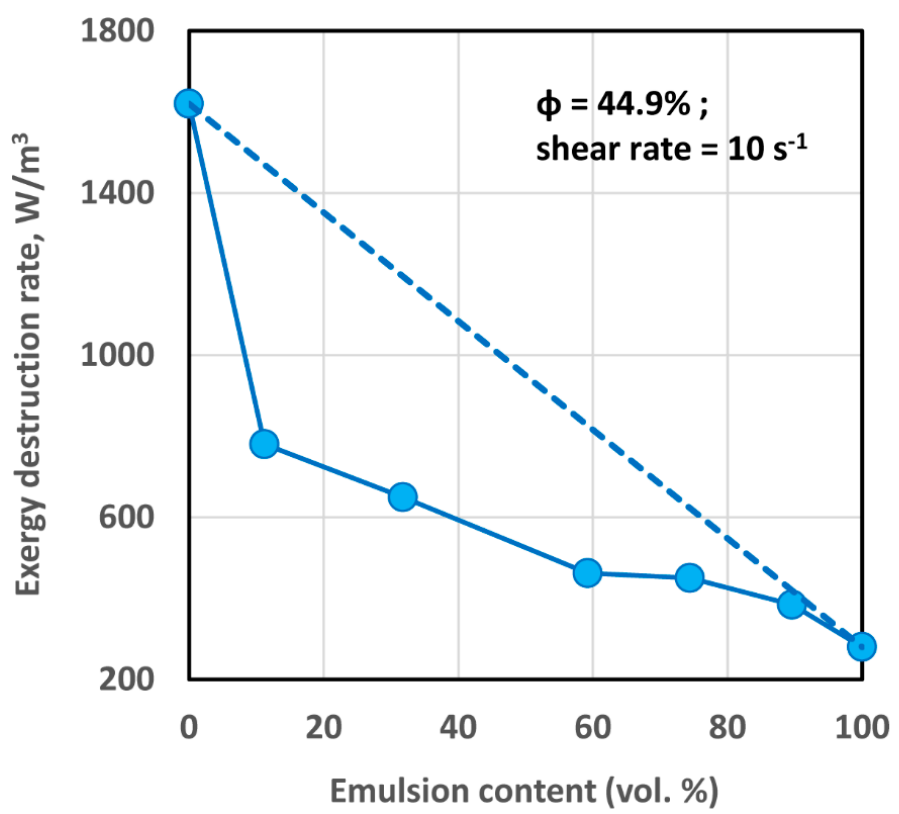

Figure 12. Exergy destruction in blends of emulsion and suspension as a function of emulsion content ( $\mathrm{vol} \%$ ) at a constant total volume fraction of dispersed phase of 0.449 and shear rate of $10 \mathrm{~s}^{-1}$.

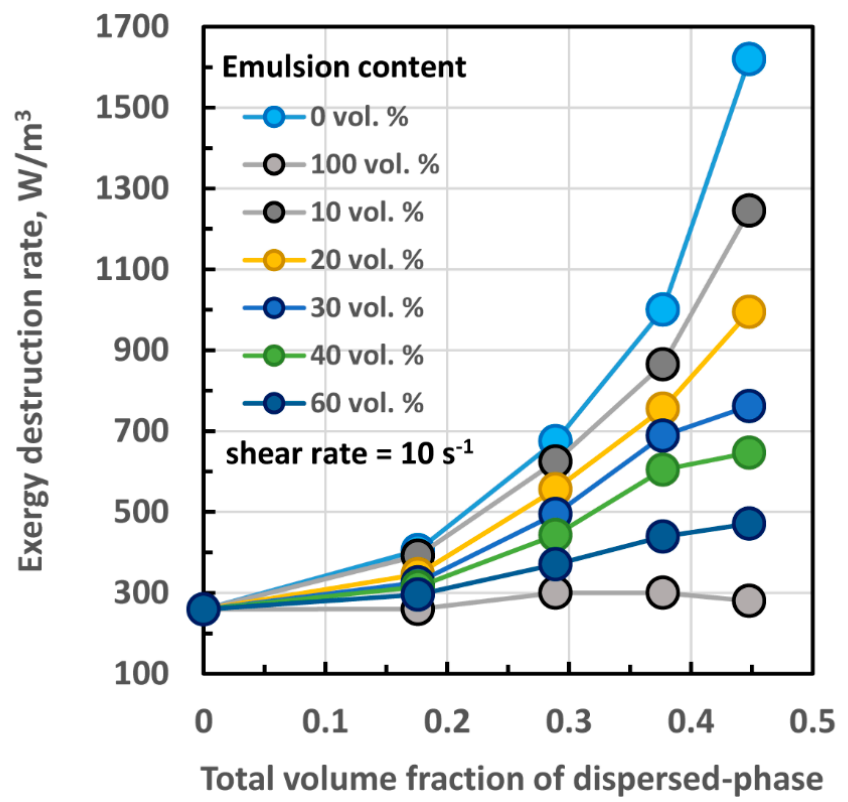

Figure 13. Exergy destruction in blends of emulsion and suspension as a function of total volume fraction of dispersed phase for different contents of emulsion at a shear rate of $10 \mathrm{~s}^{-1}$.

\subsubsection{Model for Calculation of Exergy Destruction in Multi-Phase Dispersions}

The droplets of emulsion are much smaller in size as compared with the solid particles (glass beads) of suspension. The Sauter mean diameter of solid particles is approximately 27 times larger than that of emulsion droplets. Furthermore, the rheology of emulsions is not significantly different than that of the polymer matrix alone. Thus, it is reasonable to treat oil droplets and polymeric matrix together as an effective matrix for the solid particles. This is shown schematically in Figure 14. The three-phase system of oil droplets/glass beads/polymeric matrix is equivalent to a two-phase system consisting of solid particles dispersed in an effective matrix of polymeric liquid and oil droplets. Based on this reasoning, it is expected that the relative exergy destruction in three-phase blends is a unique function 
of the concentration (volume fraction) of solid particles alone, where the relative exergy destruction is defined as a ratio of the exergy destruction rate in three-phase blend to exergy destruction rate in the effective matrix composed of oil droplets and polymeric liquid (that is, the emulsion phase).

Three-phase mixture

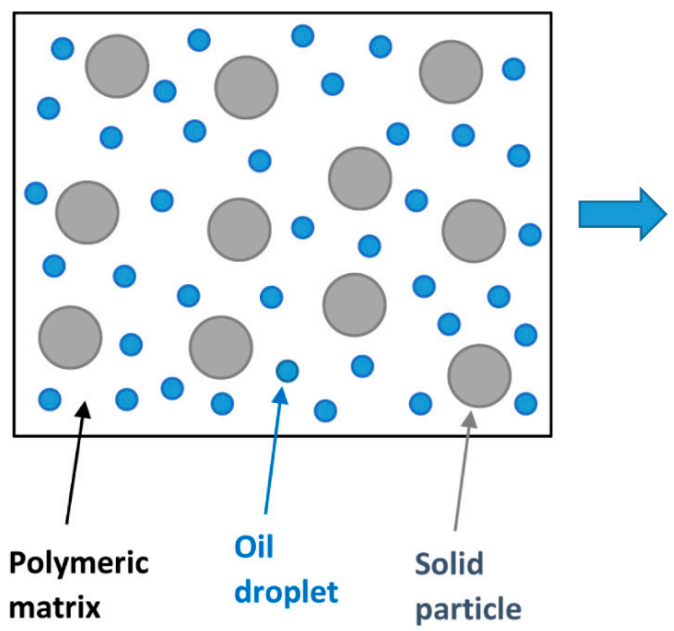

Equivalent two-phase system

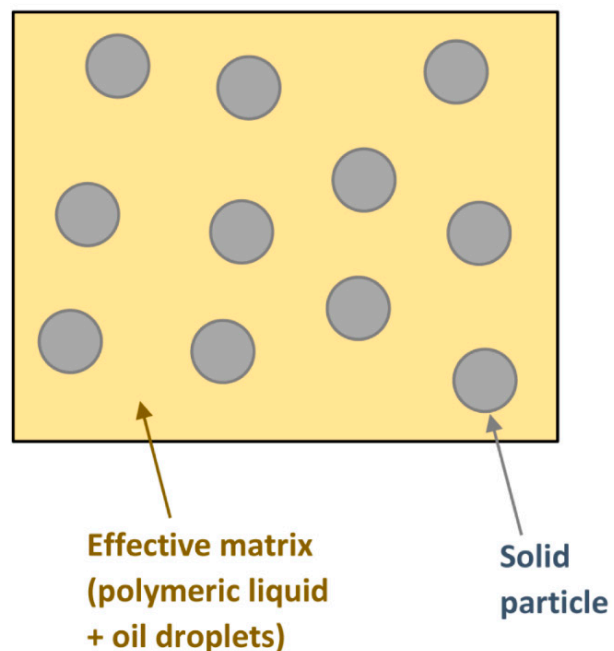

Figure 14. Three-phase blend of oil droplets, solid particles, and polymeric liquid can be treated as equivalent to a two-phase system consisting of solid particles dispersed in an effective matrix composed of oil droplets and polymeric liquid, that is, the emulsion phase.

The experimental data of Figure 13 are re-plotted in Figure 15 as relative exergy destruction rate versus volume fraction of solid (glass beads) particles in the blend. Clearly all the data of three-phase blends with different emulsion contents fall on the same curve. Also note that the pure suspension data, with polymeric liquid as the matrix, also fall on the same curve. The relative exergy destruction data can be described by the following model, shown as solid curve in Figure 15:

$$
\dot{\psi}_{D, r}=\frac{\dot{\psi}_{D, \text { blend }}}{\dot{\psi}_{D, \text { emulsion }}}=\left(1-\frac{\phi_{S}}{\phi_{m}}\right)^{-2.5 \phi_{m}}
$$

where $\phi_{S}$ is the volume fraction of solid particles in the blend, and $\phi_{m}$ is the maximum packing volume fraction of solid particles where jamming of suspension takes place. In the model prediction curve shown in Figure 15, $\phi_{m}$ is taken to be 0.64 . At $\phi_{m}$, the suspension viscosity, and consequently, the relative exergy destruction rate, is expected to diverge. The value of $\phi_{m}$ where jamming of particles, and hence, divergence of suspension viscosity takes place, is still an open question in the literature. However, a large number of experimental studies [21-26] available on the viscosity of suspensions of spherical particles have used $\phi_{m}$ of 0.64 to successfully correlate the relative viscosity versus particle concentration data. It should be noted that $\phi_{m}$ of 0.64 corresponds to random close packing of uniform spheres. As discussed in Section 4.1.2, the exergy destruction rate in emulsions can be expressed as

$$
\dot{\psi}_{D, \text { emulsion }}=K_{e} \dot{\gamma}^{n+1}
$$

where $K_{e}$ is the consistency index of emulsion and $n$ is the flow behavior index of emulsion. From Equations (37) and (38), it follows that

$$
\dot{\psi}_{D, b l e n d}=K_{e} \dot{\gamma}^{n+1}\left(1-\frac{\phi_{S}}{\phi_{m}}\right)^{-2.5 \phi_{m}}
$$


This equation can be used to calculate the exergy destruction rate in three-phase blends of this study at any given shear rate, and volume fraction of solid particles in the blend. Note that for the system under consideration, $K_{e}=6.84 \mathrm{~Pa}_{\mathrm{s}}{ }^{n} ; n=0.52$; and $\phi_{m}=0.64$.

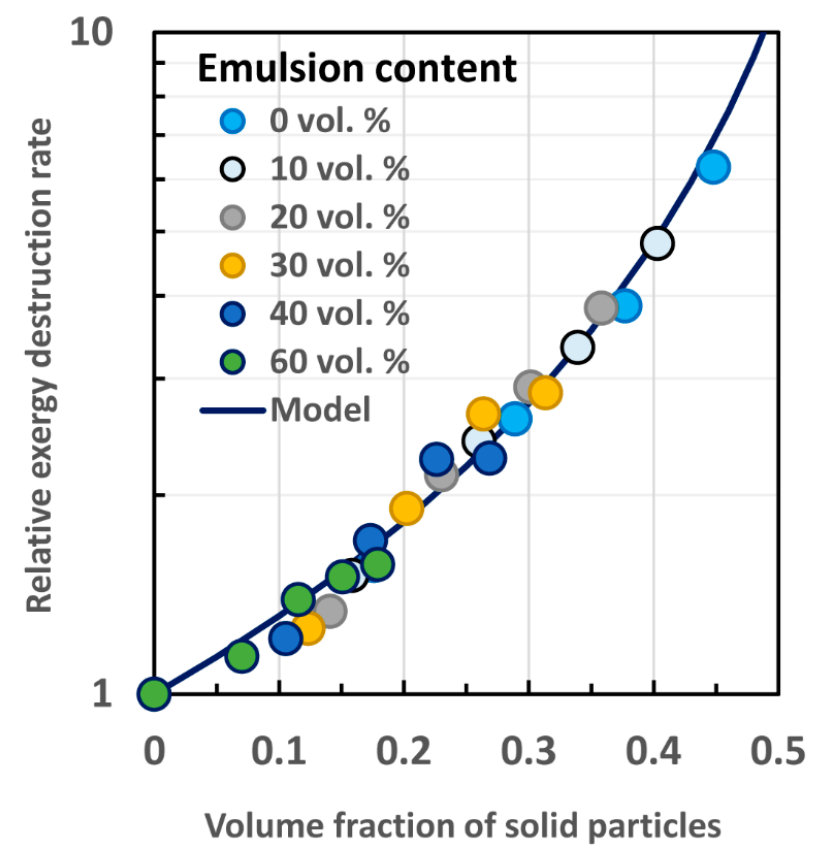

Figure 15. Relative exergy destruction rate in three-phase blend of oil droplets, solid particles, and polymeric liquid.

\section{Practical Implications of This Work}

The rate of exergy destruction is a measure of level of irreversibility in the process. According to the Gouy-Stodola theorem, the power loss in a process due to irreversibilities is equal to the rate of exergy destruction in the process. Thus, the mechanical energy dissipation rate (power loss) due to frictional effects in fluid flow can be quantified in terms of the exergy destruction rate. For a finite control volume, the global rate of exergy destruction can be calculated once the local rate of exergy destruction is known, using the following integration:

$$
\dot{\Psi}_{D, C V}=\int_{C V} \dot{\psi}_{D, l o c a l} d V
$$

where $\dot{\Psi}_{D, C V}$ is the global or overall rate of exergy destruction in the finite control volume, and $\dot{\psi}_{D, l o c a l}$ is the local rate of exergy destruction per unit volume of the fluid. In the present work, the local rate of exergy destruction in three-phase blends is given by Equation (39). Thus,

$$
\begin{gathered}
\dot{\Psi}_{D, C V}=\int_{C V} K_{e} \dot{\gamma}^{n+1}\left(1-\frac{\phi_{S}}{\phi_{m}}\right)^{-2.5 \phi_{m}} d V \\
=K_{e}\left(1-\frac{\phi_{S}}{\phi_{m}}\right)^{-2.5 \phi_{m}} \int_{C V} \dot{\gamma}^{n+1} d V
\end{gathered}
$$

The constant terms are taken outside the integral. As the shear rate may vary with position in the control volume, it is kept inside the integral. From the knowledge of the velocity field, the local shear rate and its variation inside the control volume can be calculated. Hence, the global or overall rate of exergy destruction can be determined for a given finite control volume using Equation (41). 


\section{Conclusions}

Based on the experimental work and analysis, the following conclusions can be made:

(a) The addition of oil droplets to a polymeric matrix does not alter the consistency index and flow behavior index of the system to any appreciable extent.

(b) The addition of solid particles to a polymeric matrix does not alter the flow behavior index significantly, but the consistency index increases sharply with the increase in the particle concentration.

(c) The exergy destruction rate in flow of emulsions of oil droplets and polymeric matrix does not change to any appreciable extent with the increase in the concentration of oil droplets.

(d) The exergy destruction rate in flow of suspensions of solid particles and polymeric matrix increases with the increase in particle concentration.

(e) Three-phase blends of oil droplets, solid particles, and polymeric matrix, can be modelled as two-phase blends of solid particles dispersed in an effective matrix composed of oil droplets and polymeric matrix.

(f) A model is proposed to estimate the exergy destruction rates in flow of three-phase dispersions of oil droplets, solid particles, and polymeric matrix.

Conflicts of Interest: The author declares no conflict of interest.

\section{References}

1. Conley, R.F. Practical Dispersion; VCH: New York, NY, USA, 1996.

2. Tadros, T.F. Solid/Liquid Dispersions; Academic Press: London, UK, 1987.

3. Bennett, H.; Bishop, J.L.; Wulfinghoff, M.F. Practical Emulsions-Applications; Chemical Publishing: New York, NY, USA, 1968.

4. Lissant, K.J. Emulsions and Emulsion Technology; Dekker: New York, NY, USA, 1974.

5. Torrey, S. Emulsions and Emulsifier Applications-Recent Developments; Noyes Data Corporation: Park Ridge, NJ, USA, 1984.

6. Friberg, S. Food Emulsions; Dekker: Rumson, NJ, USA, 1976.

7. Becher, P. Encyclopedia of Emulsion Technology; Dekker: New York, NY, USA, 1985; Volume 2.

8. Han, C.D. Multiphase Flow in Polymer Processing; Academic Press: New York, NY, USA, 1981.

9. Kissa, E. Dispersions: Characterization, Testing, and Measurement; Dekker: New York, NY, USA, 1999.

10. Pal, R. Rheology of emulsions containing polymeric liquids. In Encyclopedia of Emulsion Technology; Becher, P., Ed.; Dekker: New York, NY, USA, 1996; Volume 4, pp. 93-263.

11. Pal, R. Quantification of irreversibilities in practical cyclic processes using exergy analysis and Gouy-Stodola theorem. Int. J. Mech. Eng. Educ. 2017. [CrossRef]

12. Pal, R. Influence of droplet size on exergy destruction in flow of concentrated non-Newtonian emulsions. Energies 2016, 9, 293. [CrossRef]

13. Pal, R. Entropy production in pipeline flow of dispersions of water in oil. Entropy 2014, 16, 4648-4661. [CrossRef]

14. Pal, R. Exergy destruction in pipeline flow of surfactant-stabilized oil-in-water emulsions. Energies 2014, 7, 7602-7619. [CrossRef]

15. Pal, R. Entropy generation in flow of highly concentrated non-Newtonian emulsions in smooth tubes. Entropy 2014, 16, 5178-5197. [CrossRef]

16. Pal, R. Second law analysis of adiabatic and non-adiabatic pipeline flows of unstable and surfactant-stabilized emulsions. Entropy 2016, 18, 113. [CrossRef]

17. Lebon, G.; Jou, D.; Casas-Vazquez, J. Understanding Non-Equilibrium Thermodynamics; Springer: Berlin/Heidelberg, Germany, 2008.

18. Pal, R. Rheology of Particulate Dispersions and Composites; CRC Press: Boca Raton, FL, USA, 2007.

19. Bird, R.B.; Armstrong, R.C.; Hassager, O. Dynamics of Polymeric Liquids_Fluid Mechanics; Wiley \& Sons: New York, NY, USA, 1987. 
20. Pal, R. Anomalous wall effects in parallel plate torsional flow of highly concentrated emulsions. ASME FED 1999, 249, 137-150.

21. Pal, R. Modeling the viscosity of concentrated nanoemulsions and nanosuspensions. Fluids 2016, 1, 11. [CrossRef]

22. Stickel, J.J.; Powell, R.L. Fluid mechanics and rheology of dense suspensions. Annu. Rev. Fluid Mech. 2005, 37, 129-149. [CrossRef]

23. Van der Werf, J.C.; De Kruif, C.G. Hard-sphere colloidal dispersions: The scaling of rheological properties with particle size, volume fraction, and shear rate. J. Rheol. 1989, 33, 421-454. [CrossRef]

24. Shikata, T.; Pearson, D.S. Viscoelastic behavior of concentrated spherical suspensions. J. Rheol. 1994, $38,601$. [CrossRef]

25. Cheng, Z.D.; Zhu, J.X.; Chaikin, P.M.; Phan, S.E.; Russel, W.B. Nature of the divergence in low shear viscosity of colloidal hard-sphere dispersions. Phys. Rev. E 2002, 65, 041405. [CrossRef] [PubMed]

26. Mewis, J.; Wagner, N.J. Colloidal Suspension Rheology; Cambridge University Press: Cambridge, UK, 2012.

(C) 2018 by the author. Licensee MDPI, Basel, Switzerland. This article is an open access article distributed under the terms and conditions of the Creative Commons Attribution (CC BY) license (http:/ / creativecommons.org/licenses/by/4.0/). 